\title{
Downregulation of microRNA-142-3p and its tumor suppressor role in gastric cancer
}

\author{
YI WANG $^{1}$, ZHIDONG CAO $^{2,3}$, LANLAN WANG $^{4}$, SIQI LIU $^{4}$ and JIANQIANG CAI ${ }^{5}$ \\ ${ }^{1}$ Department of VIP, National Cancer Center/Cancer Hospital, Chinese Academy of Medical Sciences (CAMS) \\ and Peking Union Medical College (PUMC), Beijing 100021; ${ }^{2}$ Department of Orthopedics, \\ The First Affiliated Hospital of Chongqing Medical University, Chongqing 400016; ${ }^{3}$ Department of Orthopedics, \\ The Emergency Medical Center of Chongqing, Chongqing 400014; ${ }^{4}$ Institute of Basic Medical Sciences, \\ Chinese Academy of Medical Sciences (CAMS) and Peking Union Medical College (PUMC), Beijing 100005; \\ ${ }^{5}$ Department of Hepatobiliary Surgery, National Cancer Center/Cancer Hospital, Chinese Academy of \\ Medical Sciences (CAMS) and Peking Union Medical College (PUMC), Beijing 100021, P.R. China
}

Received December 15, 2015; Accepted February 23, 2018

DOI: $10.3892 / 01.2018 .8330$

\begin{abstract}
An increasing number of studies indicate that microRNAs (miRNAs) may exert an oncogenic or tumor suppressive role in diverse types of cancer. MicroRNA (miR)-142-3p has been detected to be downregulated in a number of cancer types, and it may function as a tumor suppressor. However, the expression profile and potential role of miR-142-3p in gastric cancer remain unknown. In the present study, the expression of miR-142-3p in numerous gastric cancer samples was investigated. It was observed that miR-142-3p was markedly downregulated in cancer tissues compared with normal tissues. Furthermore, a low expression level of miR-142-3p was associated with higher tumor stages. The overexpression of miR-142-3p was able to inhibit the proliferation, invasion and migration of gastric cancer cells. A further investigation into the mechanism underlying the effect of miR-142-3p identified cyclin T2 (CCNT2) as a target of miR-142-3p in gastric cancers. miR-142-3p may exert its tumor suppressor role partially by downregulating CCNT2. These results suggested that the abnormal downregulation of miR-142-3p and the subsequent increase in CCNT2 expression may have an important role in gastric cancer carcinogenesis.
\end{abstract}

Correspondence to: Dr Jianqiang Cai, Department of Hepatobiliary Surgery, National Cancer Center/Cancer Hospital, Chinese Academy of Medical Sciences (CAMS) and Peking Union Medical College (PUMC), 17 Paniiayuan Nanli, Beijing 100021, P.R. China

E-mail: caijianqiang188@sina.com

Key words: gastric cancer, microRNA-142-3p, cyclin T2, tumor suppressor

\section{Introduction}

Gastric cancer is the third most common cause of cancer-associated mortalities in the world $(1,2)$. The treatment methods for gastric cancer primarily include surgery, radiotherapy and chemotherapy $(3,4)$. However, many patients with gastric cancer often present with advanced stages of the disease at initial diagnosis. Therefore, the overall therapeutic effect of advanced cancer remains poor $(5,6)$. The discovery of new biomarkers for earlier stages of gastric cancer and testing the sensitivity of these biomarkers is urgently required for early detection of gastric cancer and for improving therapy.

miRNAs are endogenous small non-coding RNA molecules, which have critical roles in multiple biological processes by regulating mRNAs via cleavage or inhibiting translation. A number of properties of microRNAs (miRNAs/miRs) make them attractive as potential biomarkers and therapy targets. An increasing number of studies report that miRNAs have regulatory roles in a diverse range of biological processes and that aberrant expression of miRNAs is involved in numerous diseases (7). miRNAs have been reported to act as oncogenes or tumor suppressors in a variety of types of cancer, including lung, pancreatic, breast, hepatic cancer and gastric cancer (8-13). The deregulation of many miRNAs have been detected in gastric cancer, including miR-125b, miR-124 and the miR-106b-25 and miR-221-222 clusters (14). Furthermore, several miRNAs have critical roles in gastric cancer carcinogenesis and the expression levels of these miRNAs may predict the outcome of patients with gastric cancer (15). Consequently, miRNAs may be important biomarkers for the diagnosis and prevention of gastric cancer.

miR-142-3p has been reported to be downregulated in diverse types of cancer, including leukemia, thyroid follicular carcinomas, cervical cancer, hepatic cancer, glioblastoma, osteosarcoma and non-small cell lung cancer (16-22). miR-142-3p has been demonstrated to contribute to carcinogenesis by regulating cell cycle, cell migration, apoptosis and invasion by targeting various signaling pathways and 
targets $(19,20,23)$. However, aberrant miR-142-3p expression in gastric cancer and the potential role of miR-142-3p in gastric cancer carcinogenesis are largely uninvestigated.

In the present study, TaqMan probes were employed to analyze miR-142-3p expression in 100 pairs of gastric cancer tissues and the adjacent normal tissues. The results indicated that miR-142-3p was markedly downregulated in gastric cancer tissues compared with adjacent non-neoplastic tissues. In addition, a lower level of miR-142-3p expression in gastric cancer was significantly associated with higher tumor stages, which indicated its tumor inhibitory role in gastric cancer carcinogenesis. Furthermore, the overexpression of miR-142-3p was able to inhibit the proliferation, invasion and migration of gastric cancer cells, and these effects may be mediated via downregulating cyclin $\mathrm{T} 2$, which is a regulator of cell cycle.

\section{Materials and methods}

Patients and specimens. The human gastric cancer and corresponding adjacent non-neoplastic tissues were collected from surgical specimens of 100 patients with gastric cancer at the VIP Department, National Cancer Center, Cancer Hospital, Chinese Academy of Medical Sciences (Beijing, China) between January 2015 and December 2015. The sex ratio of the patients and controls was 1:1 and the age range was 30 to 70 years old. Overall staging grouping outlined by AJCC, also referred to as Roman numeral staging which uses numerals I, II, III and IV, was used to describe the progression of cancer (24). The present study was approved by the Ethics Committee of the Department of VIP, National Cancer Center/Cancer Hospital, Chinese Academy of Medical Sciences. The tumor and non-cancerous tissues were histologically confirmed by $\mathrm{H} \& \mathrm{E}$ staining. Briefly, paraffin-embedded tissues were sliced into 4- $\mu \mathrm{M}$-thick sections and pretreated at $65^{\circ} \mathrm{C}$ for $2 \mathrm{~h}$, followed by deparaffinization using xylene performed twice, for $10 \mathrm{~min}$ each. Subsequently the tissues were rehydrated in absolute alcohol twice, for $5 \mathrm{~min}$ each, followed by $95 \%$ ethanol for $2 \mathrm{~min}$ and $70 \%$ ethanol for $2 \mathrm{~min}$. The sections were washed briefly in distilled water twice and stained using Harris hematoxylin solution for $8 \mathrm{~min}$ at room temperature followed by washing in running tap water for $5 \mathrm{~min}$, then differentiating in $1 \%$ acid ethanol for $30 \mathrm{sec}$. Bluing in $0.2 \%$ ammonia water or saturated lithium carbonate solution for $30 \mathrm{sec}$ to $1 \mathrm{~min}$ at room temperature was conducted followed by washing in running tap water for $5 \mathrm{~min}$. Finally, the sections were dehydrated using $95 \%$ ethanol, followed by absolute ethanol twice for $5 \mathrm{~min}$ each, followed by xylene twice for $5 \mathrm{~min}$ each and mounted with a xylene based mounting medium. The sections were photographed under a light microscope (Nikon TE2000, Japan) equipped with a digital camera. All clinical samples were snapped frozen in liquid nitrogen immediately and stored at $-80^{\circ} \mathrm{C}$ until RNA extraction.

Cell culture and transfection. The human gastric cancer cell lines HGC-27, MGC-803 and 293T cells (American Type Culture Collection, Manassas, VA, USA) were cultured in Dulbecco's modified Eagle's medium (Invitrogen; Thermo Fisher Scientific, Inc., Waltham, MA, USA) supplemented with $10 \%$ fetal bovine serum (FBS) at $37^{\circ} \mathrm{C}$ in $5 \% \mathrm{CO}_{2}$. Scrambled control mimic (cat. no. CN-001000-01-05), miR-142-3p mimics (cat. no. MIMAT0000434), cyclin T2 (CCNT2) small-interfering siRNA (cat. no. L-003221-00-0005) and siRNA control (cat. no. D-001810-01-05) were purchased from GE Healthcare Dharmacon, Inc., (Lafayette, CO, USA) and transfected into HGC-27, MGC-803 and 293T cells at $50 \mathrm{nM}$ using DharmFECT1 (GE Healthcare Dharmacon, Inc.).

RNA extraction, cDNA synthesis and reverse transcriptionquantitative polymerase chain reaction ( $R T-q P C R)$ assays. Total RNA was extracted using Trizol from human tissues and gastric cancer cells according to the manufacturer's instructions. cDNA was synthesized using 1-5 $\mu \mathrm{g}$ total RNA and a high-capacity cDNA reverse transcription kit (Thermo Fisher Scientific, Inc.), according to the manufacturer's instructions. For detection of miR-142-3p expression, a miR-142-3p specific RT primer was used for reverse transcription. RT-qPCR using SYBR-Green qPCR Master mix (Takara Bio, Inc., Otsu, Japan) was performed in a Bio-Rad CFX96 real-time PCR system using TaqMan probes with U6 small nuclear RNA as an endogenous control. The PCR parameters were as follows: $95^{\circ} \mathrm{C}$ for $30 \mathrm{sec}$, followed by 40 cycles of $95^{\circ} \mathrm{C}$ for $5 \mathrm{sec}$ and $60^{\circ} \mathrm{C}$ for $34 \mathrm{sec}$. The relative expression of miRNAs and coding genes was calculated using the $2^{-\Delta \Delta \mathrm{Cq}}$ method (25). The primer sequences used in the present study are listed in Table I.

Cell proliferation and colony formation assay. To determine the possible effect of miRNA mimics or CCNT2 siRNA on cell proliferation, the transfected gastric cancer cells were incubated with $10 \%$ Cell Counting Kit-8 (CCK-8) (Dojindo Molecular Technologies, Inc., Kumamoto, Japan) at $37^{\circ} \mathrm{C}$ for $1 \mathrm{~h}$ and analyzed at $450 \mathrm{~nm}$. Growth curve of gastric cancer cells were constructed by detecting absorbance at day 1, 2, 3 and 4 post-transfection. Subsequently, the colony formation ability was detected. Gastric cancer cells were trypsinized and replated at 200 cells per well in 6-well plates, maintained for a week and then stained with $0.1 \%$ crystal violet in $20 \%$ methanol for $15 \mathrm{~min}$. The number of colonies was counted., and was performed. All the experiments were repeated three times. The aforementioned control mimic and siRNA control were used as negative controls for miRNA mimic and CCNT2 siRNA, respectively.

Cell migration and invasion assays. To determine the migratory ability of gastric cancer cells, artificial scratches were created by tips $24 \mathrm{~h}$ after transfection. The images were captured under a light microscope (Nikon TE2000; Nikon Corporation, Tokyo, Japan) equipped with a digital camera at 0,24 and $48 \mathrm{~h}$ following the scratches, and the percentage of open wound area was calculated [(open wound area/initial wound area) x100].

For the invasion assay, $1 \times 10^{5}$ gastric cancer cells were plated onto a Matrigel-coated Transwell chamber and inserted in wells of a 24-well plate in serum-free DMEM medium. In addition, $10 \%$ FBS was added below the chamber as a chemoattractant. After culturing at $37^{\circ} \mathrm{C}$ for $24 \mathrm{~h}$, the cells that invaded the lower surface of the chamber were stained with $0.1 \%$ crystal violet for $10 \mathrm{~min}$ at room temperature and washed with PBS and counted under a microscope (Nikon TE2000; Nikon Corporation,) equipped with a digital camera. 
Table I. Sequence of primers used in reverse transcriptionquantitative polymerase chain reaction and constructs.

\begin{tabular}{ll}
\hline Primer & \multicolumn{1}{c}{ Sequence (5'-3') } \\
\hline $\begin{array}{l}\text { miR-142-3p } \\
\text { RT }\end{array}$ & GTCGTATCCAGTGCAGGGTCCGAGGTA \\
& TTCGCACTGGATACGACTCCATAA \\
Forward & CTGTGTAGTGTTTCCTACTTTA \\
Reverse & GTGCAGGGTCCGAGGT \\
Probe & FAM-GTAGTGTTTCCTACTTTATGG-MGB \\
U6 & \\
RT & AAAATATGGAACGCTTCACGAATTTG \\
Forward & CTCGCTTCGGCAGCACATATACT \\
Reverse & ACGCTTCACGAATTTGCGTGTC \\
Probe & FAM-CCATGCTAATCTTCTCTGTA-MGB \\
CCNT2-UTR & \\
Forward & ACCAGATTGGCTGCTGAA \\
Reverse & CCCTACAAAGAGGCTCCTAAGT \\
\hline
\end{tabular}

CCNT2, cyclin T2; miRNA, microRNA; RT, reverse transcription; UTR, untranslated region.

Constructs and luciferase assay. A number of potential targets of miR-142-3p were predicted using online microRNA target predicting tools: PicTar (http://www.pictar.org/) and TargetScan (http://www.targetscan.org/vert_71/). The 3'untranslated region (UTR) of human CCNT2 containing miR-142-3p binding sites was inserted downstream of the firefly luciferase reporter in the pMIR-reporter (Promega Corporation, Madison, WI, USA). Mutations at the miRNA binding site in the 3'UTR sequence were created using bridging PCR and then the mutated CCNT2 3'-UTR was also cloned into the pMIR-reporter. For luciferase assay, $0.4 \mu \mathrm{g}$ reporter construct, $0.02 \mu \mathrm{g}$ pRL-TK vector and 5 pmol miRNA mimic or scrambled controls were co-transfected using Lipofectamine 2000 (Invitrogen; Thermo Fisher Scientific, Inc.) into $293 \mathrm{~T}$ cells. The cells were harvested and lysed $48 \mathrm{~h}$ post-transfection, the luciferase activity was detected using a dual luciferase assay (Promega Coporation) in triplicate. The relative luciferase activity was normalized with Renilla luciferase activity and a pMIR-reporter containing a complete complementary sequence to miR-142-3p as positive control.

Western blotting. The whole-cell lysate of MGC-803 cells and HGC-27 cells was extracted with the lysis buffer (0.05 M Tris, $\mathrm{pH}, 7.5,0.15 \mathrm{M} \mathrm{NaCl}$ and 2\% NP-40) containing $200 \mathrm{mM}$ $\mathrm{NaF}, 200 \mathrm{~lm} \mathrm{Na} \mathrm{VO}_{4}, 0.5 \mathrm{M}$ EDTA and proteinase inhibitors, for $30 \mathrm{~min}$ on ice and then quantified using a BCA protein assay kit. $10 \mu \mathrm{g}$ protein lysates were then separated by $12 \%$ SDS-PAGE and transferred onto a polyvinylidene difluoride membrane for the detection of CCNT2 and GAPDH. The membraned were blocked in 5\% skim milk. The following antibodies were used at a 1:1,000 dilution: Anti-CCNT2 (catalog no. 21860-1-AP; ProteinTech Group, Inc., Chicago, IL, USA) and anti-GAPDH (catalog no. 10494-1-AP; ProteinTech Group, Inc.). Horseradish peroxidase-conjugated goat anti-rabbit IgG secondary antibody (catalog no. SA00001-1; ProteinTech Group, Inc.) was used at a 1:10,000 dilution and $5 \%$ milk was used as blocking reagent. Immobilon Western HRP substrate (Merck KGaA, Darmstadt, Germany) was used as a visualization reagent.

Statistical analysis. Spearman's correlation was used to analyze the association between clinicopathological characteristics and miR-142-3p expression. A Student's t-test (two-tailed) was used to analyze the data. All statistical analysis was conducted using SPSS 19.0 (IBM Corp., Armonk, NY, USA). Data are presented as the mean \pm standard deviation. $\mathrm{P} \leq 0.05$ was considered to indicate a statistically significant difference.

\section{Results}

miR-142-3p is significantly downregulated in gastric cancer tissues. To investigate the expression of miR-142-3p in gastric cancer, miR-142-3p expression was examined in 100 pairs of gastric cancer tissues and the adjacent normal tissues by qPCR using TaqMan probes. The threshold for miR-142-3p expression change was set as \pm 1.5 . The results indicated that miR-142-3p expression was decreased in $57 \%(57 / 100)$ of cancer tissues compared with the adjacent non-neoplastic tissues in patients with gastric cancer (Fig. 1A). miR-142-3p expression was upregulated in 19\% (19/100) of cancer tissues compared with corresponding normal tissues in patients with gastric cancer. 24/100 (24\%) of patients exhibited no change in miR-142-3p expression (Fig. 1A). The results also indicated that miR-142-3p expression in gastric cancer samples was markedly lower compared with non-neoplastic tissues $(\mathrm{P}<0.01)$ (Fig. 1B). To further investigate the association between the clinicopathological characteristics and the expression level of miR-142-3p, the relative expression of miR-142-3p in 100 pairs of gastric cancer and normal tissues were analyzed. Clinical correlation analysis by Spearman's correlation demonstrated that a lower level of miR-142-3p expression in gastric cancer was associated with higher stages of the disease (stage I/II vs. III/IV, P<0.01; Fig. 1C). No significant associations were observed between miR-142-3p expression and sex, age, position or Borrmann typing (data not shown). The significant downregulation of miR-142-3p in gastric cancer tissues and its association with the malignant phenotype strongly indicated miR-142-3p is able to regulate the development of gastric cancer.

Overexpression of miR-142-3p is able to inhibit the growth of gastric cancer cells. To investigate the potential role of miR-142-3p in gastric cancer carcinogenesis, miR-142-3p was overexpressed in two gastric cancer cell lines: HGC-27 and MGC-803 (Fig. 2). miR-142-3p was successfully overexpressed in the two cell lines as detected by qPCR (Fig. 2A and C). CCK-8 assay was used to determine the cell proliferation rate at day 1,2, 3 and 4 post-transfection. Cell growth curve indicated that the overexpression of miR-142-3p in HGC-27 and MGC-803 cell lines was able to markedly inhibit the proliferation of cancer cells compared with the scrambled control. (Fig. 2B and D). Furthermore, the colony formation ability of miR-142-3p-overexpressed gastric cells was also examined. The transfected cells were re-plated at a relative 
A

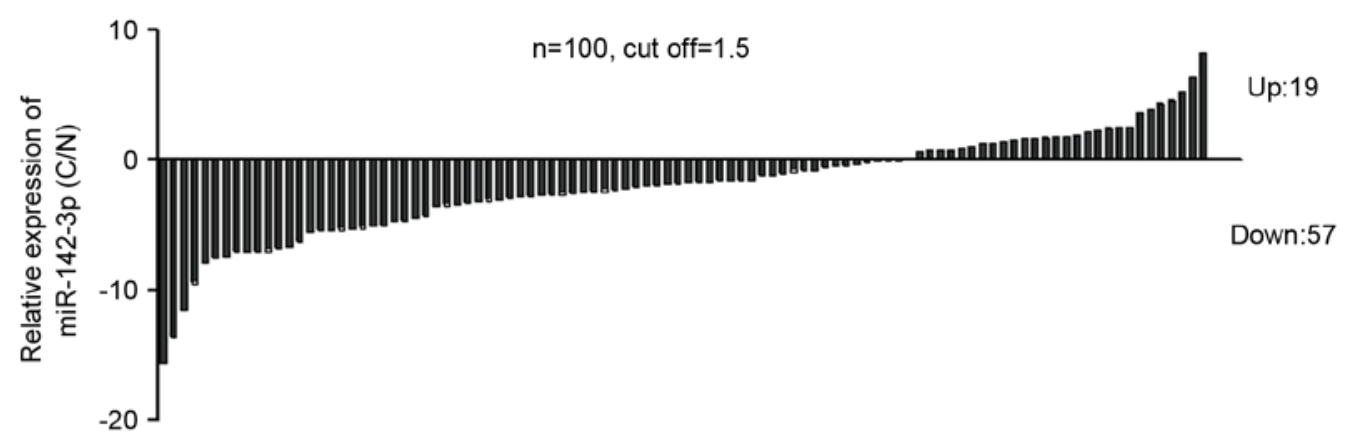

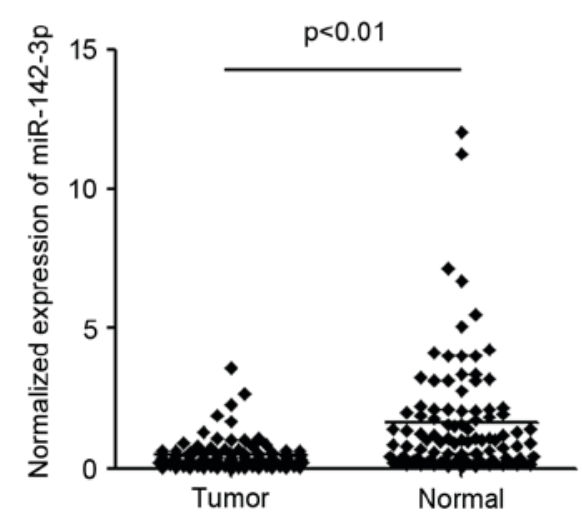

C

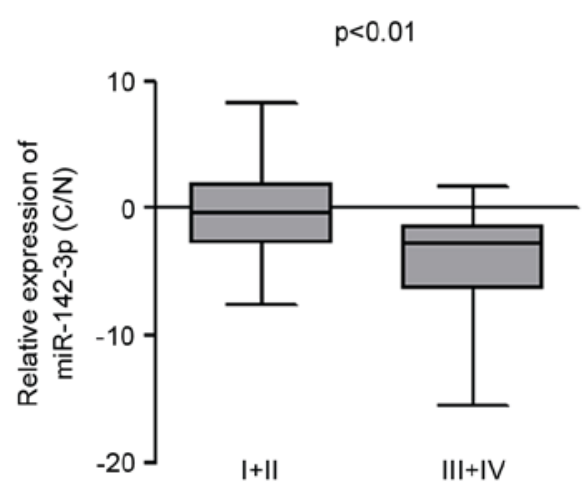

Figure 1. miR-142-3p is significantly downregulated in GC tissues. (A) The relative expression of miR-142-3p in 100 pairs of GC tissues and adjacent non-neoplastic tissues. (B) Normalized expression of miR-142-3p in 100 pairs of gastric cancer and corresponding adjacent non-neoplastic tissues. (C) The association between miR-142-3p expression and tumor stages. C, GC tissues; GC, gastric cancer; miRNA, microRNA; N, non-neoplastic tissues.

low density and maintained for 7 days to allow the colonies to form. The overexpression of miR-142-3p was able to significantly decrease the number of HGC-27 and MGC-803 colonies compared with the scrambled control. By contrast, the scrambled control exerted little effects on the number of colonies formed compared with the untreated cells (Fig. 2E and F). Collectively, the results suggested that miR-142-3p may perform a tumor suppressive role in gastric cancer.

miR-142-3p is able to suppress the migration and invasion of gastric cancer cells. To investigate whether miR-142-3p regulates the aggressive properties of gastric cancer cells, the migratory and invasive abilities of gastric cancer cells that were overexpressed with miR-142-3p were assessed. A wound-healing/scratch assay was used to evaluate the role of miR-142-3p in regulating the migration of gastric cancer cells. The overexpression of miR-142-3p was able to decrease the migration rate in the two gastric cancer cell lines (Fig. 3A and B). Wound healing was markedly decreased in miR-142-3p mimic-transfected gastric cancer cells at different time points compared with the scrambled control (Fig. 3A and B). In addition, a Matrigel cell invasion assay was employed to investigate the potential effect of miR-142-3p on the invasive ability of gastric cancer cells. The overexpression of miR-142-3p was able to significantly decrease the number of invaded cells compared with the scrambled control and untreated cells (Fig. 3C and D). These results suggested that miR-142-3p is able to regulate the migration and invasion of gastric cancer cells.
CCNT2 is a direct target of miR-142-3p in gastric cancer cells. The mechanism by which miR-142-3p regulates the growth and migration of gastric cancer cells was further examined. A number of potential targets of miR-142-3p were predicted using PicTar and TargetScan.

In particular, CCNT2 was identified as a potential candidate target. The 3'-UTR of CCNT2 contains a sequence that matches with the 'seed' sequence of miR-142-3p (Fig. 4A). CCNT2 is a member of highly conserved cyclin family, which regulates cyclin-dependent kinase (CDK) kinase activity, and it is expressed periodically during cell cycle. CCNT2 and its kinase partner CDK9 were identified to be components of the transcription elongation factor complex, $\mathrm{p}-\mathrm{TEFb}$, which was reported to facilitate transcription through phosphorylating the C-terminus of the large subunit of RNA polymerase II (26-28).

To validate whether miR-124-3p is able to regulate CCNT2, the 3'-UTR of CCNT2 (containing the miR-124-3p binding site) was cloned into a luciferase reporter construct (pMIR-reporter). The complete complementary sequence of miR-142-3p was also cloned into the reporter and used as a positive control. The luciferase activity of wild-type CCNT2 3'-UTR was downregulated by $50 \%$ when miR-142-3p mimic was transfected compared with the scrambled control (Fig. 4B). To test whether the effect was dependent on the predicted miRNA binding site, the mutated CCNT2 3'-UTR reporter was constructed, where the predicted miRNA binding site was altered. The transfection of the mutated CCNT2 3'-UTR reporter was able to eliminate the reduction in luciferase 
A

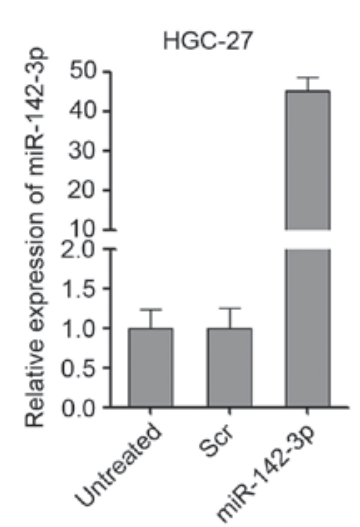

C

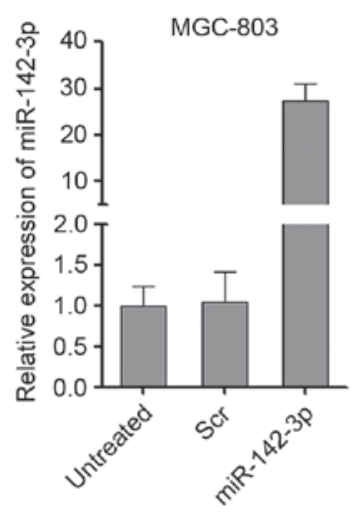

E

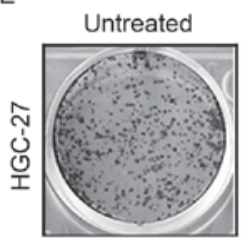

$\mathrm{F}$

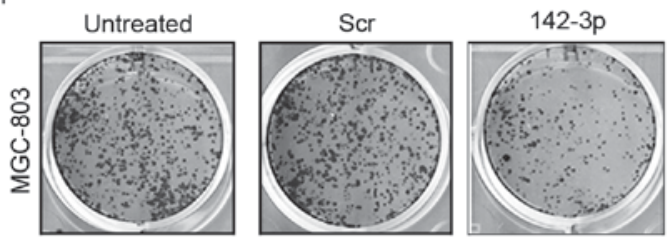

B

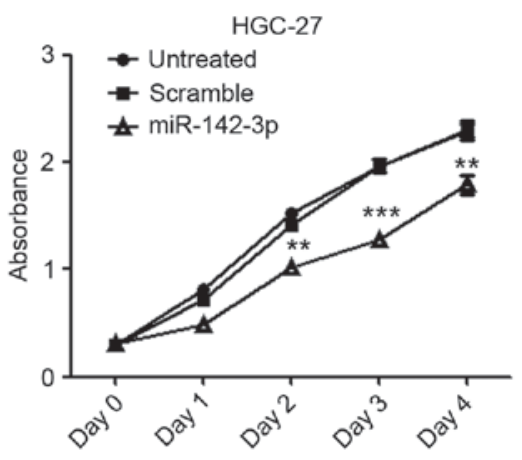

D

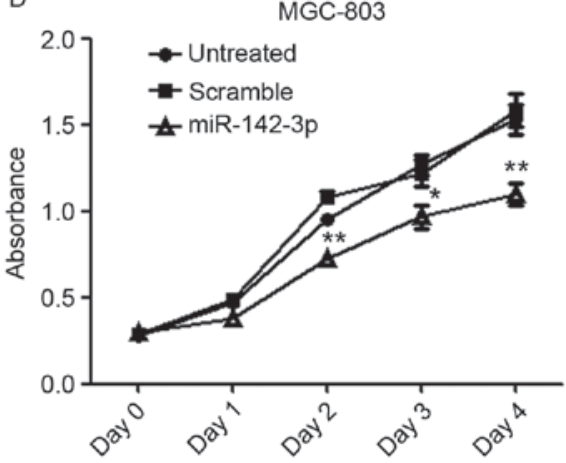

Figure 2. miR-142-3p is able to suppress the ability of the gastric cancer cells to proliferate and form colonies. (A) The enforced expression of miR-142-3p in HGC-27 cells was confirmed by RT-qPCR. (B) CCK-8 proliferation assays performed at day 0, 1, 2, 3 and 4 post-transfection of HGC-27 cells. Data are expressed as the mean \pm standard deviation $(n=3)$. (C) The enforced expression of miR-142-3p in MGC-803 cells was confirmed by RT-qPCR. (D) CCK-8 proliferation assays performed at day $0,1,2,3$ and 4 post-transfection of MGC-803 cells. Data are expressed as the mean \pm standard deviation ( $=3$ ). The number of colonies/well in 6-well plates: (E) HGC-27 and (F) MGC-803. The cells were cultured for 7 days. ${ }^{*} \mathrm{P}<0.05 ;{ }^{* *} \mathrm{P}<0.01$, ${ }^{* * *} \mathrm{P}<0.001$ vs. scrambled control. CCK-8, Cell Counting Kit-8; miRNA, microRNA; RT-qPCR, reverse transcription-quantitative polymerase chain reaction. Scr, scrambled control; 142-3p, miR-142-3p mimic.

activity, which suggests that the inhibitory effect of miR-142-3p on the CCNT2 3'-UTR was dependent on the miRNA binding site (Fig. 4C). To further validate that CCNT2 is a real target of miR-142-3p, CCNT2 protein expression was quantified by western blotting. A marked decrease in the level of CCNT2 expression was observed in HGC-27 and MGC-803 cells that were transfected with the miR-142-3p mimic compared with the cells that were transfected with the scrambled control, which supported the hypothesis that CCNT2 is a direct target of miR-142-3p (Fig. 4D).
Knock down of CCNT2 suppress the growth and invasion of gastric cancer cells. The p-TEFb complex, which contains CCNT2 and its kinase partner CDK9, has been demonstrated to negatively regulate human immunodeficiency virus type 1 Tat expression (29). CCNT2 was also reported to be able to inhibit monocytic differentiation by increasing proliferation (22). However, the role of CCNT2 in regulating gastric cancer carcinogenesis was largely unknown. To investigate the potential role of CCNT2 in gastric cancer, CCNT2 expression was knocked down using siRNA in HGC-27 and 
A

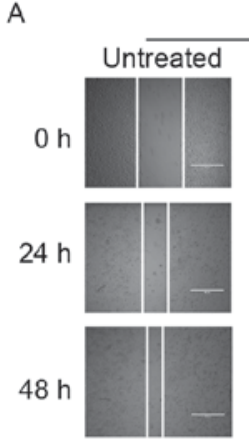

B

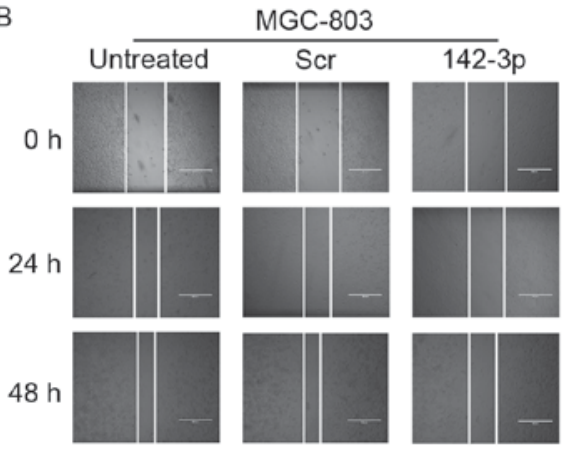

C
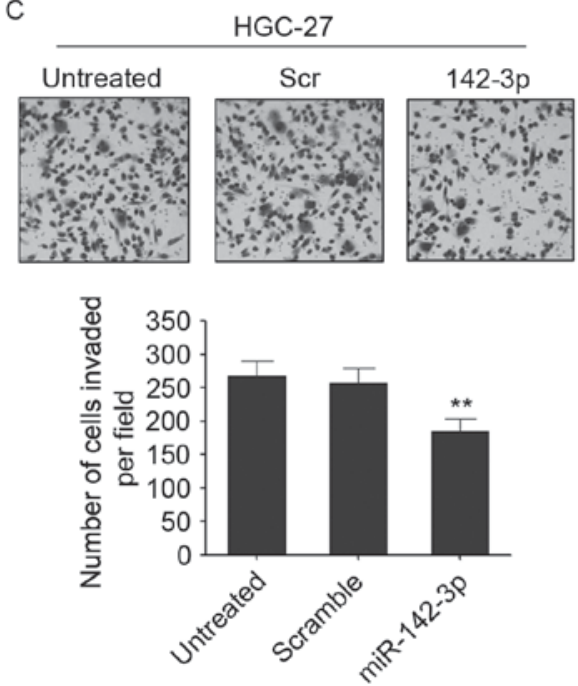
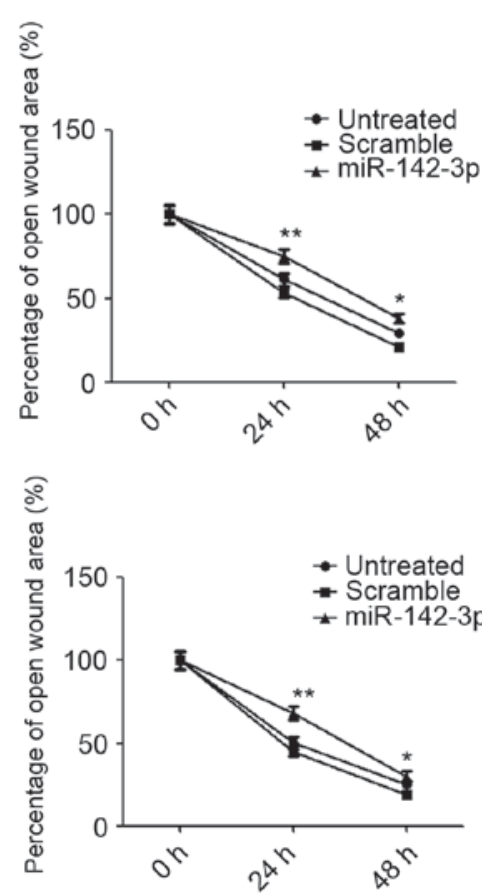

D
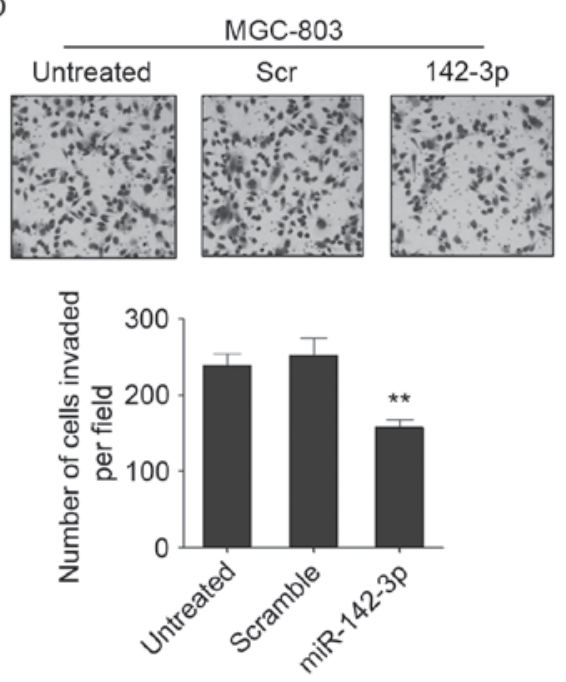

Figure 3. miR-142-3p is able to inhibit the migration and invasion of gastric cancer cells. (A) The images of the wound healing assay of cells at $0,24,48 \mathrm{~h}$ after the scratches were made: (A) HGC-27 and (B) MGC-803 cells. Data are expressed as the mean \pm standard deviation (n=3). Scale bar in A and B=500 $\mu \mathrm{m}$. The invaded cells in the Matrigel Transwell invasion assay: (C) HGC-27 and (D) MGC-803 cells. Magnification, x40. Data are expressed as the mean \pm standard deviation $(\mathrm{n}=3) .{ }^{*} \mathrm{P}<0.05 ;{ }^{* *} \mathrm{P}<0.01$ vs. scrambled control. miRNA, microRNA; Scr, scrambled control; 142-3p, miR-142-3p mimic.

MGC-803 gastric cancer cell lines. A decreased expression of CCNT2 was observed in CCNT2 siRNA-transfected gastric cancer cells compared with cells that were transfected with siRNA control and untreated cells (Fig. 5A). The knockdown of CCNT2 was able to significantly attenuate the proliferation of GC cells in the two gastric cancer cell lines (Fig. 5B). By contrast, transection of the control siRNA exerted no effects on cell proliferation compared with untreated cells (Fig. 5B). The effect of CCNT2 on cell invasion was also examined. CCNT2 knockdown was able to significantly inhibit the number of invaded cells compared with the siRNA negative control and untreated cells in HGC-27 and MGC-803 cell lines (Fig. 5C and D). These results demonstrated that $\mathrm{CCNT} 2$ might act as an oncogene in gastric cancer and the role of miR-142-3p in regulating gastric cancer cell proliferation, migration and invasion was through targeting CCNT2.

\section{Discussion}

In the present study, miR-142-3p expression was examined in patients with gastric cancer, and the potential role and mechanism of miR-142-3p in regulating GC carcinogenesis was investigated. The expression profiles of miR-142-3p indicated that miR-142-3p was significantly downregulated in GC tissues compared with adjacent non-neoplastic tissues. Furthermore, a lower level of miR-142-3p expression was associated with higher tumor stages. The overexpression of miR-142-3p was able to the proliferation, migration and invasion of gastric cancer cells. These results suggested that miR-142-3p may act 
A

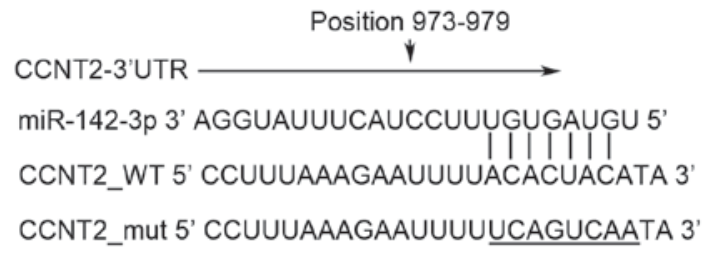

C

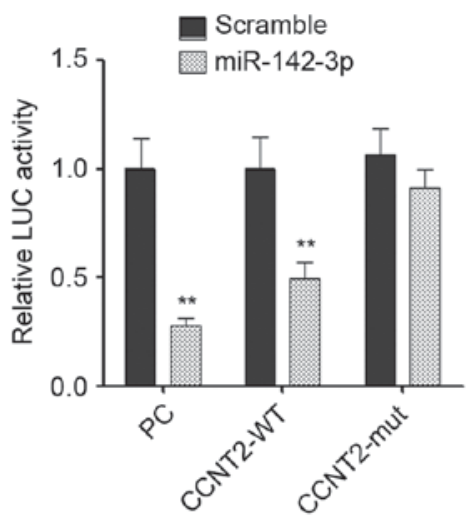

D
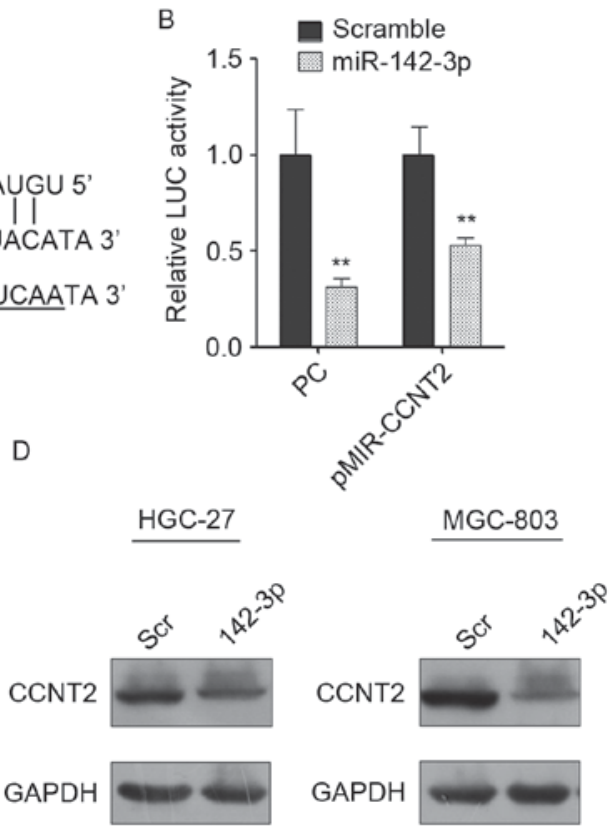

Figure 4. CCNT2 is a direct target of miR-142-3p in gastric cancer cells. (A) Prediction of binding site of miR-142-3p in CCNT2 by Pictar. (B) Relative luciferase activity of $293 \mathrm{~T}$ cells that were co-transfected with reporter constructs and miR-142-3p mimics or scrambled control. Data are expressed as the mean \pm standard deviation $(n=3)$. (C) Relative luciferase activity of 293T cells that were transfected with wild-type and mutated CCNT2 3'UTR constructs. Data are expressed as the mean \pm standard deviation $(n=3)$. ${ }^{* *} \mathrm{P}<0.01$ vs. scrambled control. (D) Western blot analysis of CCNT2 protein levels in HGC-27 and MGC-803 cells that were transfected with scrambled control or miR-142-3p mimics. CCNT2, cyclin T2; LUC, luciferase; miRNA, microRNA; mut, mutated; PC, positive control; Scr, scrambled control; UTR, untranslated region; WT, wild-type; 142-3p, miR-142-3p mimic.

as a tumor suppressor in gastric cancer, and therefore might be a potential diagnostic marker or therapeutic target for gastric cancer.

miR-142-3p has been reported to be downregulated in diverse types of cancer and to contribute to carcinogenesis. For example, downregulation of miR-142-3p was observed in a large number of follicular thyroid adenomas and carcinomas, and miR-142-3p was also reported to function as a tumor suppressor in follicular thyroid tumorigenesis (16). miR-142-3p has also been reported to be able to inhibit the proliferation and invasion of cervical cancer cells by targeting frizzled-7 (30). Furthermore, lower miR-142-3p expression in hepatic cancer was associated with poorer survival. miR-142-3p was reported to negatively regulate CD133, which is a hepatic cancer stem cell marker: $(23,31)$. In non-small cell lung cancer, miR-142-3p is able to repress transforming growth factor (TGF)- $\beta$-induced growth inhibition through inhibiting TGF $\beta$ receptor 1 (20). In human acute lymphoblastic leukemia, miR-142-3p was reported to inhibit cell proliferation by targeting the MLL-AF4 oncogene (21). In colon cancer cells, miR-142-3p was also reported to function as a tumor suppressor by targeting CD133, ATP-binding cassette sub-family $\mathrm{G}$ member 2 and leucine-rich repeat-containing G-protein coupled receptor 5 (23).

However, the aberrant expression of miR-142-3p in gastric cancer and its potential role in gastric carcinogenesis was largely unknown; there were few reports investigating the aberrant expression of miR-142-3p in gastric cancer tissues. Inoue et al (32) examined the expression level of several miRNAs in 5 patients with gastric cancer and observed that
miR-142-3p was upregulated in tumor tissues compared with normal tissues. However, the study used a small number of clinical samples, which would not reflect the real expression and regulatory role of miR-142-3p in gastric cancer. In the present study, TaqMan probe-based qPCR was employed to examine the expression level of miR-142-3p in 100 pairs of gastric cancer tissues and the corresponding adjacent normal tissues. miR-142-3p expression was detected to be significantly decreased in gastric cancer tissues compared with normal tissues. To the best of our knowledge, this is the first study to report on the aberrant downregulation of miR-142-3p in a large number of gastric cancer tissues. The inhibitory role of miR-142-3p on the proliferation, migration and invasion of GC cells was also examined, which supports the view that miR-142-3p has a tumor suppressor role in GC carcinogenesis.

Investigating the tumor suppressor role of miR-142-3p in $\mathrm{GC}$ in vivo will be the future direction of the present authors. As a lower expression of miR-142-3p was associated with a higher tumor stage, the potential therapeutic and diagnostic roles of miR-142-3p are to be examined further in a larger number of clinical specimens.

miRNAs regulate gene expression at the transcriptional and post-transcriptional levels by binding to target mRNAs and initiating the degradation of target mRNAs or inducing translational repression $(33,34)$. CCNT2 identified as a direct target of miR-142-3p that regulates gastric cancer carcinogenesis. CCNT2 is a component of the P-TEFb complex, which is essential for transcription initiation and elongation that is mediated by RNA polymerase II. P-TEFb complexes 
A

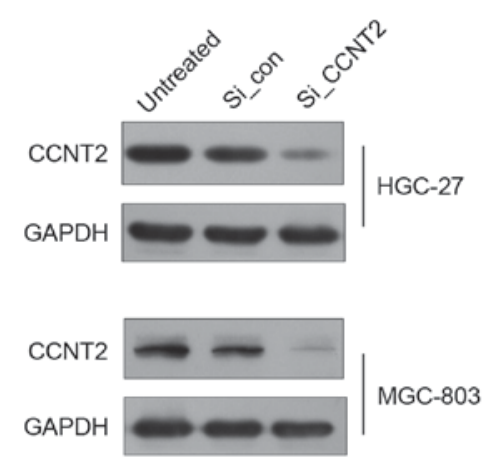

C
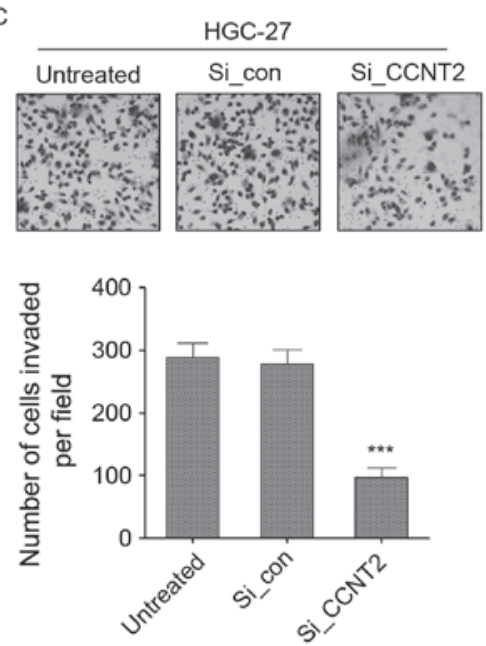

B
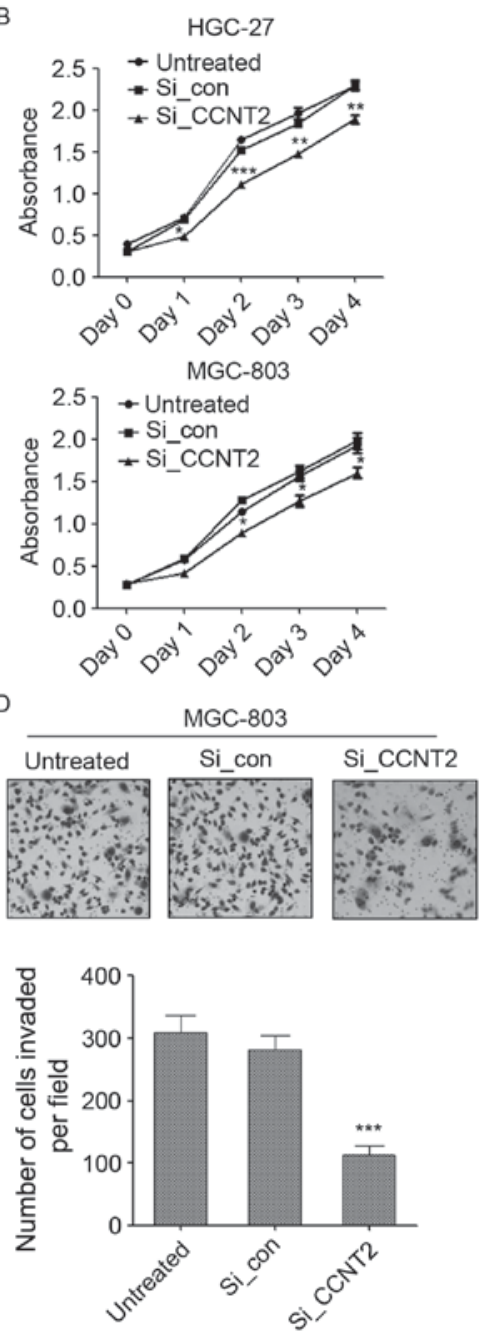

Figure 5. Knock down of CCNT2 is able to inhibit the growth and invasion of gastric cancer cells. (A) Western blot analysis of CCNT2 protein expression in HGC-27 and MGC-803 cells that were transfected with negative control or CCNT2 siRNAs. (B) CCK-8 proliferation assay performed at day 0, 1, 2, 3, 4 post-transfection of HGC-27 and MGC-803 cells. Data are expressed as the mean \pm standard deviation ( $\mathrm{n}=3$ ). Transwell Matrigel invasion assay of (C) HGC-27 and (D) MGC-803 cells. Data are expressed as the mean \pm standard deviation $(\mathrm{n}=3)$. $\mathrm{P}<0.05 ;{ }^{* *} \mathrm{P}<0.01 ;{ }^{* * * *} \mathrm{P}<0.001$ vs. scrambled control. CCK-8, Cell Counting Kit-8; CCNT2; cyclin T2; con, control; siRNA, small-interfering RNA.

have critical roles in embryonic development and multiple cellular processes $(35,36)$. The role of CCNT2 in regulating cell growth and tumorigenesis are not clear, with the exception of one study which reported that CCNT2 was able to increase the proliferation of THP- 1 cells and inhibit monocytic differentiation (22). In the present study, the role of CCNT2 in regulating the proliferation, migration and invasion of gastric cancer cells was investigated. The knockdown of CCNT2 was able to inhibit the growth, migration and invasion of gastric cancer cells, which suggests that CCNT2 has an oncogenic role in gastric cancer. Therefore, the tumor-inhibitory effect of miR-142-3p in GC needs to be validated in vivo and the detailed mechanism of CCNT2 in promoting GC cell proliferation and invasion also needs to be explored further.

Taken together, miR-142-3p is able to suppress growth, migration and invasion of gastric cancer cells by down-regulating CCNT2. The downregulation of miR-142-3p and the resulting elevated CCNT2 level may contribute to GC carcinogenesis. miR-142-3p might be a potential novel diagnostic marker or a target for the treatment of gastric cancer.

\section{Acknowledgements}

Not applicable.

\section{Funding}

The present study was supported by grants from the National High-Tech Research and Development Program of China (grant no. 2015AA020408).

\section{Availability of data and materials}

All data generated or analyzed during the present study are included in this published article.

\section{Authors' contributions}

JC and YW conceived the study. YW designed the experiments and performed the majority of them. $\mathrm{ZC}$ contributed to the performance of experiments. LW and SL performed cell culture and collected clinical samples. YW wrote the manuscript. 


\section{Ethics approval and consent to participate}

The present study was approved by the Ethics Committees of Department of VIP, National Cancer Center/Cancer Hospital, Chinese Academy of Medical Sciences (Beijing, China) and written informed consent was obtained from all patients.

\section{Consent for publication}

Written informed consent was obtained from all examined patients for the publication of their data.

\section{Competing interests}

The authors declare that they have no competing interests.

\section{References}

1. Sitarz R, Skierucha M, Mielko J, Offerhaus GJA, Maciejewski R and Polkowski WP: Gastric cancer: Epidemiology, prevention, classification, and treatment. Cancer Manag Res 10: 239-248, 2018.

2. Fock KM: Review article: The epidemiology and prevention of gastric cancer. Aliment Pharmacol Ther 40: 250-260, 2014.

3. Ang TL and Fock KM: Clinical epidemiology of gastric cancer. Singapore Med J 55: 621-628, 2014.

4. Jang JY and Chun HJ: Efficacy of Helicobacter pylori eradication for the prevention of metachronous gastric cancer after endoscopic resection for early gastric cancer. World J Gastroenterol 20: 2760-2764, 2014.

5. Meyer HJ and Wilke H: Treatment strategies in gastric cancer. Dtsch Arztebl Int 108: 698-705, 2011.

6. Peters MD: Postsurgical chemotherapy vs. surgery alone for resectable gastric cancer. Am J Nurs 114: 24, 2014.

7. Wu WK, Lee CW, Cho CH, Fan D, Wu K, Yu J and Sung JJ: MicroRNA dysregulation in gastric cancer: A new player enters the game. Oncogene 29: 5761-5771, 2010.

8. Li Y, Fang Y, Liu Y and Yang X: MicroRNAs in ovarian function and disorders. J Ovarian Res 8: 51, 2015.

9. Zhu D, Chen H, Yang X, Chen W, Wang L, Xu J and Yu L: miR-32 functions as a tumor suppressor and directly targets SOX9 in human non-small cell lung cancer. Onco Targets Ther 8: 1773-1783, 2015.

10. Lee EJ, Gusev Y, Jiang J, Nuovo GJ, Lerner MR, Frankel WL, Morgan DL, Postier RG, Brackett DJ and Schmittgen TD: Expression profiling identifies microRNA signature in pancreatic cancer. Int J Cancer 120: 1046-1054, 2007.

11. Murakami Y, Yasuda T, Saigo K, Urashima T, Toyoda H, Okanoue T and Shimotohno K: Comprehensive analysis of microRNA expression patterns in hepatocellular carcinoma and non-tumorous tissues. Oncogene 25: 2537-2545, 2006.

12. Yu S, Lu Z, Liu C, Meng Y, Ma Y, Zhao W, Liu J, Yu J and Chen J: miRNA-96 suppresses KRAS and functions as a tumor suppressor gene in pancreatic cancer. Cancer Res 70: 6015-6025, 2010.

13. Liu C, Yu J, Yu S, Lavker RM, Cai L, Liu W, Yang K, He X and Chen S: MicroRNA-21 acts as an oncomir through multiple targets in human hepatocellular carcinoma. J Hepatol 53: 98-107, 2010.

14. Ventura A and Jacks T: MicroRNAs and cancer: Short RNAs go a long way. Cell 136: 586-591, 2009.

15. Hui A, How C, Ito E and Liu FF: Micro-RNAs as diagnostic or prognostic markers in human epithelial malignancies. BMC Cancer 11: 500, 2011.

16. Colamaio M, Puca F, Ragozzino E, Gemei M, Decaussin-Petrucci M, Aiello C, Bastos AU, Federico A, Chiappetta G, Del Vecchio L, et al: miR-142-3p downregulation contributes to thyroid follicular tumorigenesis by targeting ASH1L and MLL1. J Clin Endocrinol Metab 100: E59-E69, 2015.
17. Chai S, Tong M, Ng KY, Kwan PS, Chan YP, Fung TM, Wong N, Xie D, Yuan YF, Guan XY and Ma S: Regulatory role of miR-142-3p on the functional hepatic cancer stem cell marker CD133. Oncotarget 5: 5725-5735, 2014.

18. Xu S, Wei J, Wang F, Kong LY, Ling XY, Nduom E, Gabrusiewicz K, Doucette T, Yang Y, Yaghi NK, et al: Effect of miR-142-3p on the M2 macrophage and therapeutic efficacy against murine glioblastoma. J Natl Cancer Inst 106: pii:dju162, 2014.

19. Xu G, Wang J, Jia Y, Shen F, Han W and Kang Y: MiR-142-3p functions as a potential tumor suppressor in human osteosarcoma by targeting HMGA1. Cell Physiol Biochem 33: 1329-1339, 2014.

20. Lei Z, Xu G, Wang L, Yang H, Liu X, Zhao J and Zhang HT: MiR-142-3p represses TGF- $\beta$-induced growth inhibition through repression of TGF $\beta R 1$ in non-small cell lung cancer. FASEB J 28: 2696-2704, 2014.

21. Dou L, Li J, Zheng D, Li Y, Gao X, Xu C, Gao L, Wang L and Yu L: MicroRNA-142-3p inhibits cell proliferation in human acute lymphoblastic leukemia by targeting the MLL-AF4 oncogene. Mol Biol Rep 40: 6811-6819, 2013.

22. Wang XS, Gong JN, Yu J, Wang F, Zhang XH, Yin XL, Tan ZQ, Luo ZM, Yang GH, Shen C and Zhang JW: MicroRNA-29a and microRNA-142-3p are regulators of myeloid differentiation and acute myeloid leukemia. Blood 119: 4992-5004, 2012.

23. Shen WW, Zeng Z, Zhu WX and Fu GH: MiR-142-3p functions as a tumor suppressor by targeting CD133, ABCG2, and Lgr5 in colon cancer cells. J Mol Med (Berl) 91: 989-1000, 2013.

24. Carlson RW, Allred DC, Anderson BO, Burstein HJ, Carter WB, Edge SB, Erban JK, Farrar WB, Goldstein LJ, Gradishar WJ, et al: Breast cancer. Clinical practice guidelines in oncology. J Natl Compr Canc Netw 7: 122-192, 2009.

25. Livak KJ and Schmittgen TD: Analysis of relative gene expression data using real-time quantitative PCR and the 2(-Delta Delta C(T)) method. Methods 25: 402-408, 2001.

26. de Falco G and Giordano A: CDK9 (PITALRE): A multifunctional multifunctional cdc2-related kinase. J Cell Physiol 177: 501-506, 1998.

27. De Luca A, Esposito V, Baldi A, Claudio PP, Fu Y, Caputi M, Pisano MM, Baldi F and Giordano A: CDC2-related kinase PITALRE phosphorylates $\mathrm{pRb}$ exclusively on serine and is widely expressed in human tissues. J Cell Physiol 172: 265-273, 1997.

28. Simone C, Bagella L, Bellan C and Giordano A: Physical interaction between $\mathrm{pRb}$ and cdk9/cyclinT2 complex. Oncogene 21: 4158-4165, 2002.

29. Napolitano G, Licciardo P, Gallo P, Majello B, Giordano A and Lania L: The CDK9-associated cyclins T1 and T2 exert opposite effects on HIV-1 Tat activity. AIDS 13: 1453-1459, 1999.

30. Deng B, Zhang Y, Zhang S, Wen F, Miao Y and Guo K: MicroRNA-142-3p inhibits cell proliferation and invasion of cervical cancer cells by targeting FZD7. Tumour Biol 36: 8065-8073, 2015.

31. Wu L, Cai C, Wang X, Liu M,Li X and Tang H: MicroRNA-142-3p, a new regulator of $\mathrm{RAC1}$, suppresses the migration and invasion of hepatocellular carcinoma cells. FEBS Lett 585: 1322-1330, 2011.

32. Inoue $\mathrm{T}$, Iinuma $\mathrm{H}$, Ogawa $\mathrm{E}$, Inaba $\mathrm{T}$ and Fukushima $\mathrm{R}$ : Clinicopathological and prognostic significance of microRNA-107 and its relationship to DICER1 mRNA expression in gastric cancer. Oncol Rep 27: 1759-1764, 2012.

33. Hammond SM: An overview of microRNAs. Adv Drug Deliv Rev 87: 3-14, 2015.

34. Krützfeldt J: Strategies to use microRNAs as therapeutic targets. Best Pract Res Clin Endocrinol Metab 30: 551-561, 2016.

35. Wei P, Gender ME, Fang SM, Fisher WH and Jones KA: A novel CDK9-associated C-type cyclin interacts directly with HIV-1 Tat and mediates its high-affinity, loop-specific binding to TAR RNA. Cell 92: 451-462, 1998.

36. Kohoutek J, Li Q, Blazek D, Luo Z, Jiang H and Peterlin BM: Cyclin T2 is essential for mouse embryogenesis. Mol Cell Biol 29: 3280-3285, 2009. 